\title{
Interceptive treatment of palatal impaction of maxillary canines with rapid maxillary expansion: A randomized clinical trial
}

\author{
Tiziano Baccetti, ${ }^{a}$ Manuela Mucedero, ${ }^{\mathrm{b}}$ Maria Leonardi, ${ }^{\mathrm{c}}$ and Paola Cozza ${ }^{\mathrm{d}}$ \\ Florence, Rome, and Catania, Italy
}

\begin{abstract}
Introduction: Our aim in this prospective randomized clinical study was to assess the prevalence rate of eruption of palatally displaced canines, diagnosed at an early developmental stage with posteroanterior radiographs and consequently treated by rapid maxillary expansion. Methods: A sample of 60 subjects in the early mixed dentition with palatally displaced canines diagnosed on posteroanterior radiographs was enrolled in the trial. Their age range at the first observation (T1) was 7.6 to 9.6 years, with a prepubertal stage of skeletal maturity (CS1 or CS2). The 60 subjects were randomly allocated to the treatment group (TG, 35 subjects) or the no-treatment group (NTG, 25 subjects). The TG was treated with a banded rapid maxillary expander; after expansion, all patients were retained with the expander in place for 6 months. Thereafter, the expander was removed, and the patients wore a retention plate at night for a year. The NTG received no treatment. All subjects were reevaluated in the early permanent dentition (T2) (postpubertal CS4). The number of dropouts was recorded. The main outcome recorded at T2 was successful or unsuccessful eruption of the maxillary permanent canines. The starting forms at T1 for measurements on posteroanterior and panoramic films were compared in the 2 groups with the Mann-Whitney $U$ test $(P<0.05)$. The prevalence rates of successful and unsuccessful treatments in the TG were compared with those in NTG with chi-square tests $(P<0.05)$. From T1 to T2, there were 3 dropouts in each group. Results: The final sample comprised 32 subjects in the TG and 22 subjects in the NTG. No statistically significant differences were found for any variable at T1. The prevalence rates of successful eruption of the maxillary canines were $65.7 \%$ (21 subjects) in the TG and $13.6 \%$ ( 3 subjects) in the NTG. The comparison was statistically significant (chi-square $=12.4 ; P<0.001)$. Subjects with palatally displaced canines in the early mixed dentition do not have transverse deficiency of the maxillary arch. Conclusions: The use of a rapid maxillary expander as an early interceptive approach is effective for increasing the rate of eruption of palatally displaced canines. (Am J Orthod Dentofacial Orthop 2009;136:657-61)
\end{abstract}

I mpaction of maxillary permanent canines occurs with a frequency of $0.2 \%$ to $2.8 \%$, and with a prevalence rate of $80 \%$ for palatally vs vestibular impacted canines. ${ }^{1,2}$ The impaction of the maxillary canine requires comprehensive orthodontic-surgical treatment. After surgical exposure of the tooth, ortho-

\footnotetext{
a Assistant professor, Department of Orthodontics, University of Florence, Florence, Italy; Thomas M. Graber Visiting Scholar, Department of Orthodontics and Pediatric Dentistry, School of Dentistry, University of Michigan, Ann Arbor.

${ }^{\mathrm{b}}$ Research fellow, Department of Orthodontics, University of Rome "Tor Vergata," Rome, Italy.

${ }^{\mathrm{c}}$ Private practice, Catania, Italy.

${ }^{\mathrm{d}}$ Professor and head, Department of Orthodontics, University of Rome "Tor Vergata," Rome, Italy.

The authors report no commercial, proprietary, or financial interest in the products or companies described in this article.

Reprint requests to: Tiziano Baccetti, Università degli Studi di Firenze, Via del Ponte di Mezzo, 46-48, 50127, Firenze, Italy; e-mail, t.baccetti@odonto.unifi.it. Submitted, January 2008; revised and accepted, March 2008. $0889-5406 / \$ 36.00$

Copyright (C) 2009 by the American Association of Orthodontists.

doi:10.1016/j.ajodo.2008.03.019
}

dontic forces are applied to bring the impacted canine into occlusion. ${ }^{3-5}$ Both the frequency and the complexity of treatment warrant extensive study of possible interceptive treatment measures aimed to prevent definitive impaction of initially displaced canines. Interceptive procedures include extraction of deciduous canines $^{6,7}$ or orthodontic treatment to maintain or increase maxillary arch length or perimeter. ${ }^{8,9}$ Clinical trials have reported spontaneous eruption of permanent canines in $78 \%$ and $62 \%{ }^{7}$ of patients after extraction of the deciduous canines. Prospective longitudinal studies by Leonardi et $\mathrm{al}^{9}$ and by Baccetti et al ${ }^{10}$ suggested that cervical pull headgear (alone or in addition to extraction of the deciduous canine) can induce successful eruption of a palatally displaced canine (PDC) in over $80 \%$ of patients (vs $50 \%$ to $65.5 \%$ of patients who had only the deciduous canine extracted).

Maxillary expansion has been proposed as an alternative interceptive treatment for impacted canines, since some authors have observed transverse maxillary deficiency in subjects with impacted canines. ${ }^{11,12} \mathrm{~A}$ 
recent study reported a significantly higher prevalence of canine impactions (53.6\%) in an experimental group of 84 patients in the mixed dentition with maxillary deficiency when compared with a control group of 100 orthodontic patients without maxillary transverse discrepancy (19\%). ${ }^{12}$ On the other hand, several researchers found normal-to-wide maxillary arches in subjects with palatally impacted canines. ${ }^{13-16}$

All interceptive approaches are based on diagnostic records from either panoramic radiographs or dental casts and are performed in the late mixed dentition. The diagnostic measurements for canine intraosseous displacement on panoramic films by Ericson and $\mathrm{Kurol}^{6}$ are valid after the age of 10 to 11 years, when initial resorption of the roots of the deciduous canines is expected in the transition to the late mixed dentition. Sambataro et $\mathrm{al}^{17}$ introduced a method of diagnostic and prognostic evaluation for PDC at an earlier age (8-9 years) based on posteroanterior (PA) cephalograms. They found that the closer the cusp of the PDC is to the midfacial line on a PA film at the age of 8 to 9 years (early mixed dentition), the higher the probability of impaction of the tooth in the permanent dentition.

Our aim in this study was to assess the prevalence rate of successful eruption of PDCs diagnosed at an early developmental stage on PA films and subsequently treated by rapid maxillary expansion (RME), when compared with untreated controls with PDC in a prospective randomized clinical trial.

\section{MATERIAL AND METHODS}

Our sample consisted of the records of patients in a randomized prospective clinical trial at the Departments of Orthodontics of the Universities of Florence and Rome (Tor Vergata) in Italy. An initial sample of 60 subjects in the early mixed dentition with either unilateral or bilateral PDC diagnosed on PA radiographs was enrolled. The criteria for inclusion in the clinical trial were white race; age at the first observation (T1) of 7.6 to 9.6 years; prepubertal stage of skeletal growth (CS 1 or CS 2) as assessed on lateral cephalograms according to the cervical vertebral maturation method of Baccetti et al; ${ }^{18}$ prediction of canine palatal impaction derived from analysis of PA films according to the method by Sambataro et $\mathrm{al}^{17}$ (score, $<-0.565$ ); a Class II or Class III tendency, or mild tooth-size/arch-size discrepancy; no previous orthodontic treatment; and no supernumerary teeth, odontomas, cysts, craniofacial malformations, or sequelae of traumatic injuries.

The 60 subjects were randomly allocated to 2 groups: the treatment group (TG; 35 subjects: 22 girls, 13 boys) or the no-treatment group (NTG; 25 subjects:
17 girls, 8 boys). Ethical approval was obtained from committees at both universities. Informed consent was signed by the parents of our subjects.

The patients in the TG were treated with a rapid maxillary expander soldered to bands placed on the second deciduous molars or the first permanent molars. Activation of the screw was continued until the palatal cusps of the maxillary posterior teeth were in contact with the buccal cusps of the mandibular posterior teeth. Once the required expansion was achieved, the expansion screw was locked in position with cold-cure acrylic or a metallic ligature. After expansion, all patients were retained with the expander in place for 6 months; then the expander was removed, and the patients wore a retention plate at night for 1 year.

The primary goal for maxillary expansion in the TG was to improve the intraosseous position of the PDC, as suggested in the literature. ${ }^{11,12}$ The subjects in the NTG received no orthodontic treatment.

PA cephalograms and panoramic radiographs of all subjects at T1 were analyzed. The following measurements on PA films were recorded according to the method of Sambataro et $\mathrm{al}^{17}$ for the prediction of maxillary canine impaction (Fig 1): (1) A3ccL/R to Cg vertical, the distance between the center of the maxillary left or right canine crown to the midsagittal plane; (2) $\mathrm{J} \mathrm{L} / \mathrm{R}$ to $\mathrm{Cg}$ vertical, the distance between the left or right jugal process and the midsagittal plane, indicating the width of the hemimaxilla; and (3) J-J, the distance between the left and right jugal processes to assess the skeletal width of the maxilla.

The following measurements proposed by Ericson and $\mathrm{Kurol}^{6}$ were made on the panoramic $\mathrm{x}$-rays (Fig 2): (1) $\alpha$ angle, the mesial inclination of the crown of the permanent canine to the midline; and (2) d distance, the distance of the cusp tip of the permanent canine from the occlusal line. These 2 measurements on panoramic films were intended to describe the intraosseous position of the canines, not to provide information about the prognosis of canine displacement, because these measurements become prognostic variables only after 10 to 11 years of age.

The following measurements proposed by Tollaro et $\mathrm{al}^{19}$ were made on the dental casts: (1) maxillary intermolar width, the distance between the central fossae of the maxillary right and left first molars; and (2) mandibular intermolar width, the distance between the tips of the distal cusps of the maxillary right and left first molars.

In subjects with normal occlusion, the distobuccal cusp of the mandibular first molar occludes with the central fossa of the maxillary first molar. Consequently, in subjects with normal occlusion, maxillary and mandibular intermolar widths are equal. A narrower 


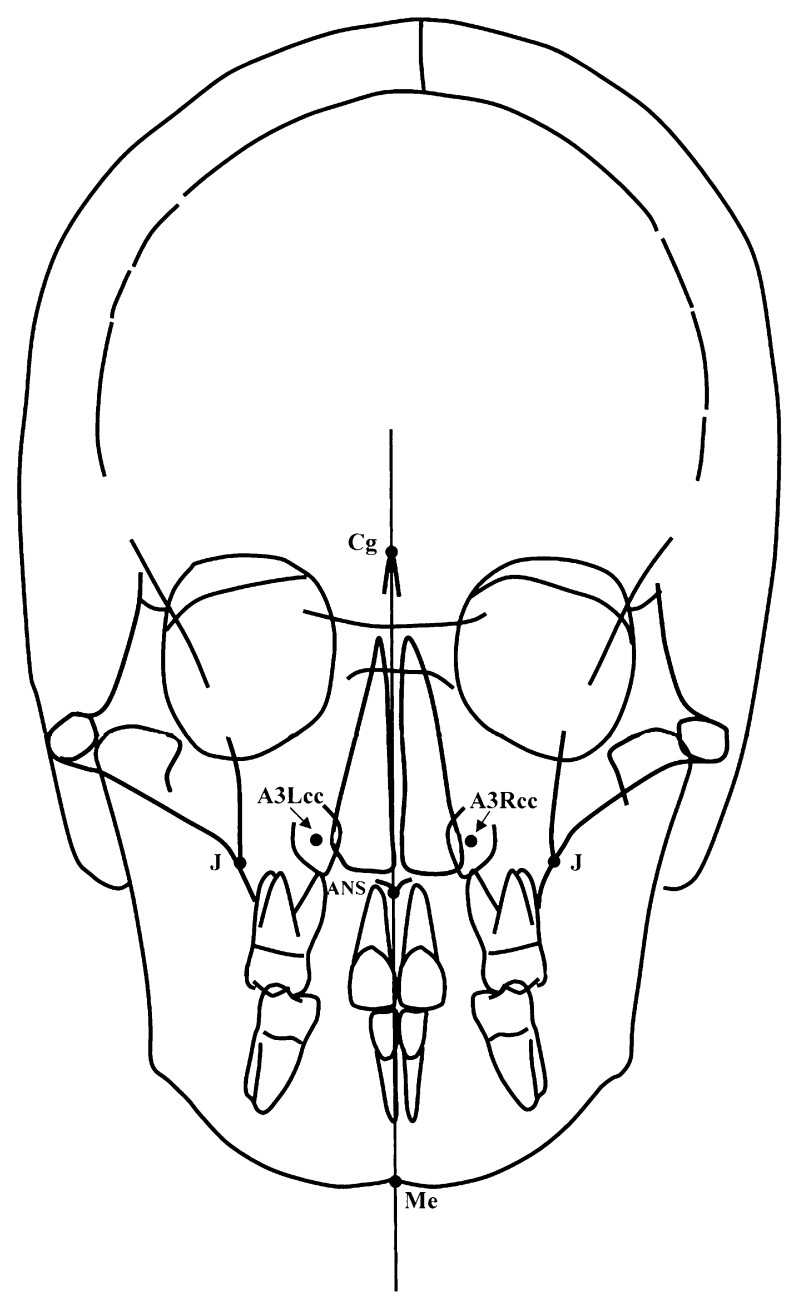

Fig 1. Landmarks and midsagittal reference line for measurements on PA films at T1.

maxillary measurement compared with the mandibular one indicates a transverse discrepancy between the dental arches. ${ }^{19}$

All subjects were reevaluated at a second observation (T2), when they were in the early permanent dentition, with a postpubertal stage of cervical vertebral maturation (CS 4). According to a recent study, the prevalence of maxillary canines that erupt spontaneously at CS 4 or after this stage of skeletal maturation is less than $6 \% .^{20}$ The average age of the patients in the TG at T2 was 13.1 years \pm 8 months, and the mean duration of observation was 4.4 years. The average age for those in the NTG at T2 was 13.3 years \pm 7 months, and the mean duration of observation was 4.11 years.

The number of dropouts was recorded. The main outcome at T2 was successful or unsuccessful eruption of the maxillary permanent canines. The successful outcome for PDC was defined as full eruption of the canine,

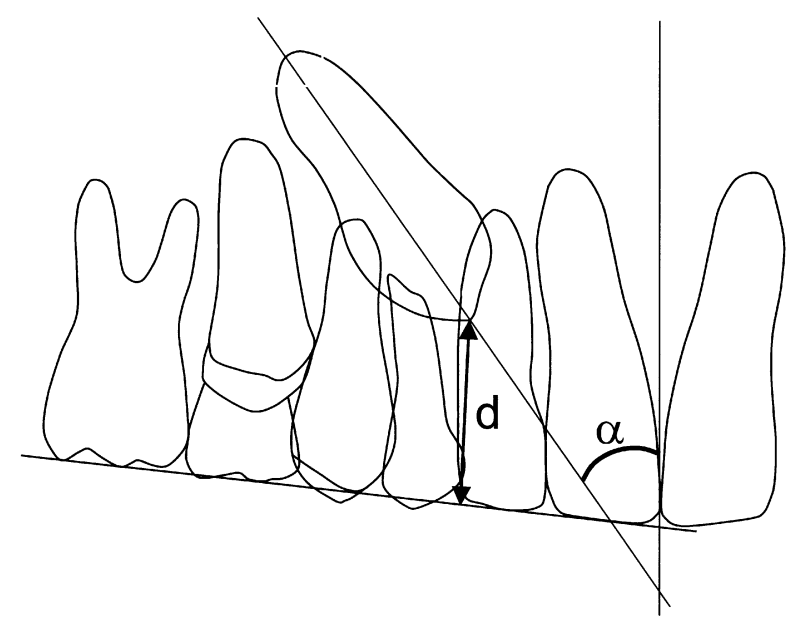

Fig 2. Measurements on panoramic radiographs at $\mathrm{T} 1$.

permitting bracket positioning for final arch alignment when needed. ${ }^{9}$ Unsuccessful outcome was a lack of eruption of the permanent canine (impaction) at $\mathrm{T} 2$.

The magnification factor for the PA films in both groups was $10 \%$. All measurements were made with the investigator (M.M.) blinded as to the group.

Method error for the cephalometric measurements on PA films was assessed with Dahlberg's formula ${ }^{21}$ on measurements repeated on 15 subjects selected randomly from the 2 groups. The measurement error for the linear measurements was on average $0.49 \mathrm{~mm}$ (range, $0.30-0.66 \mathrm{~mm}$ ). The accuracy of the measurements on the panoramic $\mathrm{x}$-rays and the dental casts was also tested by using Dahlberg's formula. ${ }^{21}$ The method errors were $1.2^{\circ}$ for $\alpha$ angle, $0.5 \mathrm{~mm}$ for the distance, and $0.4 \mathrm{~mm}$ for the 2 dental cast measurements.

\section{Statistical analysis}

The starting forms at $\mathrm{T} 1$ for measurements on the PA radiographs and panoramic films, and the dental cast measurements were compared in the TG and the NTG with the Mann-Whitney $\mathrm{U}$ test $(P<0.05)$. The prevalence rates for successful and unsuccessful patients at T2 in the TG were compared with those in the NTG with chi-square tests $(P<0.05)$.

\section{RESULTS}

There were 3 dropouts from $\mathrm{T} 1$ to $\mathrm{T} 2$ in each group. They dropped out because their families moved away during the observation period. The final sample comprised 32 subjects ( 20 girls, 12 boys; 42 PDCs) in the TG and 22 subjects (14 girls, 8 boys; 31 PDCs) in the NTG. Mean ages at T1 were 8.8 years \pm 9 months in the TG and 8.4 years \pm 1 year in the NTG. 
Table. Descriptive statistics for the measurements on PA radiographs, panoramic films, and dental casts at $\mathrm{T} 1$ in the 2 groups

\begin{tabular}{|c|c|c|c|c|c|}
\hline \multirow[b]{2}{*}{ Measurement } & \multicolumn{2}{|c|}{$T G$} & \multicolumn{2}{|c|}{$N T G$} & \multirow{2}{*}{$\begin{array}{c}\text { Mann-Whitney } \\
\text { test }\end{array}$} \\
\hline & Mean & $S D$ & Mean & $S D$ & \\
\hline $\begin{array}{l}\text { A33ccL/R-Cg vertical } \\
(\mathrm{mm})\end{array}$ & 11.1 & 0.9 & 10.3 & 1.2 & NS \\
\hline $\mathrm{J}$ L/R-Cg vertical (mm) & 30.7 & 1.8 & 31.0 & 2.2 & NS \\
\hline $\mathrm{J}-\mathrm{J}(\mathrm{mm})$ & 58.0 & 2.8 & 60.5 & 2.8 & NS \\
\hline$\alpha$ angle $\left({ }^{\circ}\right)$ & 63.6 & 6.5 & 58.6 & 7.2 & NS \\
\hline $\mathrm{d}$ distance $(\mathrm{mm})$ & 20.8 & 2.4 & 19.7 & 3.1 & NS \\
\hline $\begin{array}{l}\text { Maxillary intermolar } \\
\text { width }(\mathrm{mm})\end{array}$ & 42.1 & 2.8 & 43.2 & 2.9 & NS \\
\hline $\begin{array}{l}\text { Mandibular intermolar } \\
\text { width }(\mathrm{mm})\end{array}$ & 45.4 & 2.9 & 45.9 & 2.8 & NS \\
\hline
\end{tabular}

NS, Not significant.

The descriptive statistics for the measurements on PA radiographs, panoramic films, and dental casts at $\mathrm{T} 1$ in the groups are reported in the Table. No statistically significant differences were found for any variable.

The prevalence rates for successful eruption of the maxillary canines were $65.7 \%$ ( 21 subjects) in the TG and $13.6 \%$ (3 subjects) in the NTG. The comparison was statistically significant (chi-square $=12.382$; $P<0.001)$.

\section{DISCUSSION}

This prospective randomized clinical trial was performed to analyze the effects of RME as an early interceptive treatment modality in subjects with PDC to prevent final impaction of these teeth. The early diagnosis of PDC was made by applying the cephalometric method by Sambataro et $\mathrm{al}^{17}$ on PA films in the early mixed dentition. The successful outcome for the interceptive treatment of a PDC with RME was the full eruption of the maxillary canine in the early permanent dentition, thus allowing for bracket positioning. ${ }^{9}$

Our results indicated that subjects treated with RME have a rate for successful eruption of PDC (65.7\%) that was almost 5 times greater than that of the untreated controls $(13.6 \%)$. The difference was statistically significant $(P<0.001)$. Therefore, it can be stated that maxillary expansion is effective as an interceptive procedure to prevent final impaction of maxillary canines with palatal displacement in the early mixed dentition. The mechanism involved in the favorable outcome of RME on the eruption process of a PDC can be only speculated. It might consist of improvement in the anatomic intraosseous position of the canine because of the maxillary expansion. The analysis by Sambataro et $\mathrm{al}^{17}$ on PA films points to the reduced distance of the cusp of the canine from the midsagittal plane as a relevant predictive indicator for canine impaction.

When the pretreatment maxillary width of our subjects was contrasted with data in the literature for subjects with normal occlusal relationships, it appears that those with PDC had no transverse maxillary deficiency at the skeletal level. The average maxillary transverse dimension at the skeletal level (J-J) in subjects with normal occlusion in the mixed dentition was reported to be between 57.7 and $60.4 \mathrm{~mm}$; this is similar to the average maxillary skeletal width in the TG and the NTG in this study at T1 $(58$ and $60.5 \mathrm{~mm}$, respectively). ${ }^{14,22}$ These findings agree with the observations of several other authors. ${ }^{13-16}$ On the other hand, the transverse dimension of the maxillary arch was constricted at the dentoalveolar level. Average maxillary intermolar widths of treated and untreated subjects at $\mathrm{T} 1$ were about 42 and $43 \mathrm{~mm}$, respectively (Table). These measurements are remarkably smaller than the values reported in the literature for subjects with normal occlusal relationships $(46 \mathrm{~mm}) .{ }^{19}$ The amount of interarch transverse discrepancy at the dental level in our study (about $3.5 \mathrm{~mm}$ ) agrees with the findings of Schindel and Duffy. ${ }^{12}$ Therefore, the indication for RME in our patients was based on the palatal displacement of the canines associated with a Class II or Class III tendency, or mild tooth-size/arch-size discrepancy associated with constricted maxillary dental arches with respect to the mandibular arches.

In the subjects we examined, transverse deficiency of the maxillary arch does not relate to the etiology of the PDC, which is known to have a genetic basis. ${ }^{1}$ The transverse deficiency at the dental level was not associated with a transverse deficiency of the maxilla at the skeletal level, although it could be regarded as an occlusal sign associated with underlying Class II or Class III tendencies. ${ }^{19,22}$

The analysis of the outcomes in the NTG validates the formula proposed by Sambataro et $\mathrm{al}^{17}$ to predict the final impaction of canines displaced in an intraosseous palatal position during the early mixed dentition. The prevalence rate for canine impaction in the control group was $86.4 \%$, which was relatively similar to that in the sample examined by Sambataro et $\mathrm{al}^{17}(95.3 \%)$. On the other hand, the prevalence rate for canine impaction in the NTG of our study was greater than the rates reported by Leonardi et $\mathrm{al}^{9}(50 \%)$ and Baccetti et $\mathrm{al}^{10}$ (64\%). However, all PDCs in our NTG had the prognosis of impaction. ${ }^{17}$

The comparison of the prevalence rate for successful outcomes of RME as an interceptive procedure in subjects with PDC with those reported in previous studies on alternative treatment approaches to potentially 
impacted canines shows that RME has an effectiveness rate $(65.7 \%)$ similar to those for extraction of the deciduous canines alone $(78 \%$ according to Ericson and Kurol, ${ }^{6} 62 \%$ according to Power and Short, ${ }^{7}$ and $65.2 \%$ according to Baccetti et $\mathrm{al}^{10}$ ) or combined with fixed appliances (75\% according to Olive ${ }^{8}$ ), and smaller than the rate for eruption of the canines after use of a cervical-pull headgear $\left(87.5 \%\right.$ according Baccetti et $\left.\mathrm{al}^{10}\right)$. A main difference between these alternative interceptive approaches for a PDC and the treatment protocol investigated here is in the early timing of both diagnosis and therapy with RME, which is used in the early mixed dentition rather than the late mixed dentition.

\section{CONCLUSIONS}

The use of RME in the early mixed dentition appears to be an effective procedure to increase the rate of eruption of palatally displaced maxillary canines $(65.7 \%)$ when compared with an untreated control group.

\section{REFERENCES}

1. Peck S, Peck L, Kataja M. The palatally displaced canine as a dental anomaly of genetic origin. Angle Orthod 1994;64:249-56.

2. Bishara SE. Clinical management of impacted maxillary canines. Semin Orthod 1998;4:87-98.

3. Kohavi D, Becker A, Zilberman Y. Surgical exposure, orthodontic movement, and final tooth position as factors in periodontal breakdown of treated palatally impacted canines. Am J Orthod 1984;85: 72-7.

4. Usiskin LA. Management of the palatal ectopic and unerupted maxillary canine. Br J Orthod 1991;18:339-46.

5. Burden DJ, Mullally BH, Robinson SN. Palatally ectopic canines: closed eruption versus open eruption. Am J Orthod Dentofacial Orthop 1999;115:640-4.

6. Ericson S, Kurol J. Early treatment of palatally impacted maxillary erupting canines by extraction of the primary canines. Eur $\mathbf{J}$ Orthod 1988;10:283-95.

7. Power SM, Short MB. An investigation into the response of palatally displaced canines to the removal of deciduous canines and an assessment of factors contributing to favourable eruption. $\mathrm{Br} \mathrm{J}$ Orthod 1993;20:215-23.
8. Olive RJ. Orthodontic treatment of palatally impacted maxillary canines. Aust Orthod J 2002;18:64-70.

9. Leonardi M, Armi P, Franchi L, Baccetti T. Two interceptive approaches to palatally displaced canines: a prospective longitudinal study. Angle Orthod 2004;74:581-6.

10. Baccetti T, Leonardi M, Armi P. A randomized clinical study of two interceptive approaches to palatally displaced canines. Eur J Orthod 2008;30:381-5

11. McConnell TL, Hoffmann DL, Forbes DP, Janzen EK, Weintraub NH. Maxillary canine impaction in patients with transverse maxillar deficiency. ASDC J Dent Child 1996;63:190-5.

12. Schindel RH, Duffy SL. Maxillary transverse discrepancies and potentially impacted maxillary canines in mixed-dentition patients. Angle Orthod 2007;77:430-5.

13. Langberg BJ, Peck S. Adequacy of maxillary dental arch width in patients with palatally displaced canines. Am J Orthod Dentofacial Orthop 2000;118:220-3.

14. Cameron CG, Franchi L, Baccetti T, McNamara JA. Long-term effects of rapid maxillary expansion: a posteroanterior cephalometric evaluation. Am J Orthod Dentofacial Orthop 2002;121: 129-35.

15. Al-Nimri K, Gharaibeh T. Space conditions and dental and occlusal features in patients with palatally impacted maxillary canines: an aetiological study. Eur J Orthod 2005;27:461-5.

16. Saiar M, Rebellato J, Sheats RD. Palatal displacement of canines and maxillary skeletal width. Am J Orthod Dentofacial Orthop 2006;129:511-9.

17. Sambataro S, Baccetti T, Franchi L, Antonini F. Early predictive variables for upper canine impaction as derived from posteroanterior cephalograms. Angle Orthod 2005;75:28-34.

18. Baccetti T, Franchi L, McNamara JA Jr. The cervical vertebral maturation (CVM) method for the assessment of optimal treatment timing in dentofacial orthopedics. Semin Orthod 2005;11: 119-29.

19. Tollaro I, Baccetti T, Franchi L, Tanasescu CD. Role of posterior transverse interarch discrepancy in Class II, Division 1 malocclusion during the mixed dentition phase. Am J Orthod Dentofacial Orthop 1996;110:417-22.

20. Baccetti T, Franchi L, De Lisa S, Giuntini V. Eruption of the maxillary canines in relation to skeletal maturity. Am J Orthod Dentofacial Orthop 2008;133:748-51.

21. Dahlberg AG. Statistical methods for medical and biological students. London, United Kingdom: Bradford and Dickens; 1940.

22. Franchi L, Baccetti T. Transverse maxillary deficiency in Class II and Class III malocclusions: a cephalometric and morphometric study on postero-anterior films. Orthod Craniofac Res 2005;8: 21-8. 